This item was submitted to Loughborough's Research Repository by the author.

Items in Figshare are protected by copyright, with all rights reserved, unless otherwise indicated.

\title{
An extended adaptive Kalman filter for real-time state estimation of vehicle handling dynamics
}

PLEASE CITE THE PUBLISHED VERSION

http://www.informaworld.com/smpp/title $\sim \mathrm{db}=$ all content=g714021327

PUBLISHER

Taylor and Francis (@ Swets \& Zeitlinger)

VERSION

AM (Accepted Manuscript)

LICENCE

CC BY-NC-ND 4.0

\section{REPOSITORY RECORD}

Best, Matt C., T.J. Gordon, and P.J. Dixon. 2011. "An Extended Adaptive Kalman Filter for Real-time State Estimation of Vehicle Handling Dynamics”. figshare. https://hdl.handle.net/2134/8313. 
This item was submitted to Loughborough's Institutional Repository (https://dspace.lboro.ac.uk/) by the author and is made available under the following Creative Commons Licence conditions.

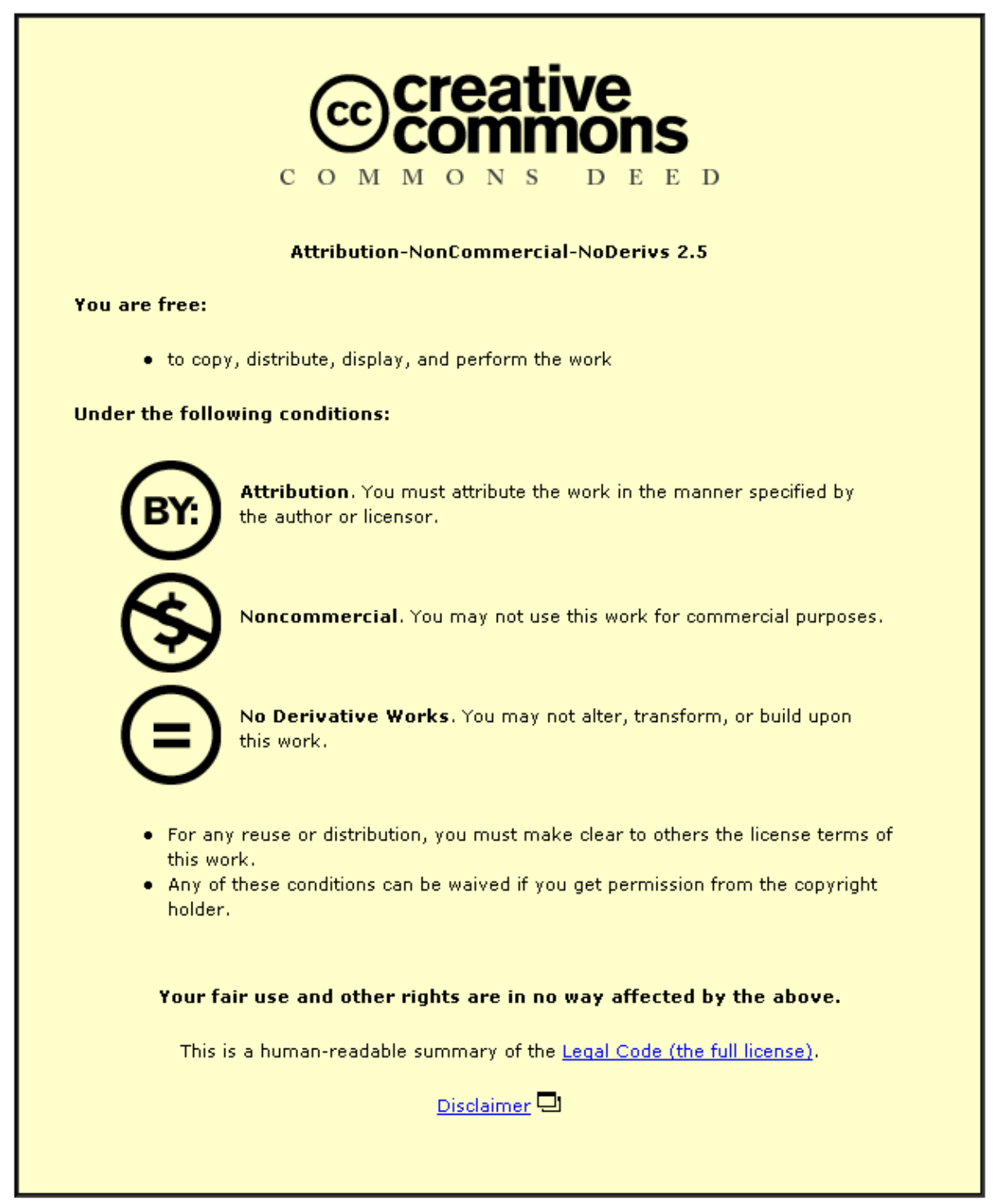

For the full text of this licence, please go to: http://creativecommons.org/licenses/by-nc-nd/2.5/ 


\title{
An Extended Adaptive Kalman Filter for Real-time State Estimation of Vehicle Handling Dynamics
}

Running header: $\quad$ An EA Kalman Filter for Handling Dynamics

\author{
M. C. BEST*, T. J. GORDON* and P. J. DIXON*
}

\section{SUMMARY}

This paper considers a method for estimating vehicle handling dynamic states in real-time, using a reduced sensor set; the information is essential for vehicle handling stability control and is also valuable in chassis design evaluation. An extended (nonlinear) Kalman filter is designed to estimate the rapidly varying handling state vector. This employs a low order (4 DOF) handling model which is augmented to include adaptive states (cornering stiffnesses) to compensate for tyre force nonlinearities. The adaptation is driven by steer-induced variations in the longitudinal vehicle acceleration.

The observer is compared with an equivalent linear, model-invariant Kalman filter. Both filters are designed and tested against data from a high order source model which simulates six degrees of freedom for the vehicle body, and employs a combined-slip Pacejka tyre model. A performance comparison is presented, which shows promising results for the extended filter, given a sensor set comprising three accelerometers only. The study also presents an insight into the effect of correlated error sources in this application, and it concludes with a discussion of the new observer's practical viability.

Keywords : Vehicle Handling Dynamics, Kalman Filter, State Observer

\section{NOTATION}

\section{Dynamic Variables}

$r \quad$ yaw rate $(\mathrm{rad} / \mathrm{s})$

$v \quad$ sideslip velocity $(\mathrm{m} / \mathrm{s})$

$u$ forward velocity $(\mathrm{m} / \mathrm{s})$

$w \quad$ wheel rotation velocity referred to tyre contact patch $(\mathrm{m} / \mathrm{s})$

$p \quad$ roll rate $(\mathrm{rad} / \mathrm{s})$

$\phi \quad$ roll angle (rad)

$\delta \quad$ (front) wheel steer angle (rad)

$\mathrm{C}_{\alpha \mathrm{f}} \mathrm{C}_{\alpha \mathrm{r}}$ front, rear cornering stiffness (N/rad)

$S_{1}-S_{5}$ acceleration sensors $\left(\mathrm{m} / \mathrm{s}^{2}\right)$

$F_{\text {lat }} F_{\text {long }}$ lateral, longitudinal components of front tyre force $(\mathrm{N})$

$\mathbf{y} \quad$ sensor set including measurement noise $(\mathrm{m} / \mathrm{s} 2)$

$\mathbf{x}^{\mathrm{s}} \quad$ state vector $-s$ superscript denotes source model data. caret denotes estimated signal

\begin{tabular}{|c|c|}
\hline \multicolumn{2}{|c|}{$\underline{\text { Model parameters }}$} \\
\hline$M$ & vehicle mass : $830 \mathrm{Kg}$ \\
\hline$I_{x x}$ & roll moment of inertia : $500 \mathrm{Kgm}^{2}$ \\
\hline$I_{z z}$ & yaw moment of inertia : $1600 \mathrm{Kgm}^{2}$ \\
\hline$I_{x z}$ & yaw/roll product of inertia : $30 \mathrm{Kgm}^{2}$ \\
\hline$h$ & height of CG above roll centre : $0.25 \mathrm{~m}$ \\
\hline$d$ & height of sensor above roll centre $: 1.0 \mathrm{~m}$ \\
\hline$b$ & CG to front axle distance : $1.06 \mathrm{~m}$ \\
\hline$c$ & CG to rear axle distance $: 1.53 \mathrm{~m}$ \\
\hline$K_{f} K_{r}$ & front, rear roll stiffness : \\
\hline & $K_{f}+K_{r}=40 \mathrm{kN} / \mathrm{rad}$ (identified) \\
\hline$B_{f} B_{r}$ & front, rear roll damping : \\
\hline & $\begin{array}{l}B_{f}+B_{r}=1.5 \mathrm{kNs} / \mathrm{rad} \text { (identified) } \\
\text { gravitational acceleration }\end{array}$ \\
\hline
\end{tabular}

* Department of Aeronautical and Automotive Engineering, Loughborough University, Loughborough, Leics. LE11 3TU, U.K 


$\begin{array}{ll}\mu & \text { longitudinal tyre friction coefficient : } \\ & 5000 \mathrm{Ns} / \mathrm{m} \\ \rho & \text { measurement noise amplification factor } \\ \lambda & \text { adaptation rate coefficient }\end{array}$

\section{$\underline{\text { Kalman Filter }}$}

$\begin{array}{ll}\omega & \text { process noise } \\ v & \text { measurement noise }\end{array}$

A,B,C,D discrete-time linear observer model

$\mathbf{K}$ Optimal gain matrix
$\mathbf{P} \quad$ state error covariance matrix

$\mathbf{R}$ measurement noise covariance matrix

$\mathbf{Q} \quad$ process noise covariance matrix

S measurement / process noise cross covariance matrix

$\mathbf{f}(\mathbf{x}) \quad$ nonlinear system model

$\mathbf{h}(\mathbf{x}) \quad$ nonlinear output model

$\mathbf{F}(\mathbf{x}) \quad$ system model Jacobian

$\mathbf{H}(\mathbf{x})$ output model Jacobian

\section{INTRODUCTION}

Recent research interest in vehicle stability has resulted in a number of proposed strategies for yaw rate, attitude and cornering control for vehicles under extreme steer manouevres and with uncertain road friction. A number of schemes have also been introduced in production vehicles - most notably by Mercedes-Benz.

Although much effort has gone into the controller designs, relatively few studies have been published in observer design. Also, while simple strategies have been suggested which require only the directly measureable yaw rate, there is an increasing need for a more complete handling state vector (eg yaw rate, sideslip velocity, roll rate and roll angle) to be available $[1,2,3]$. This data set is not directly measurable, and sideslip velocity in particular is extremely difficult to reconstruct without precise knowledge of the dynamic tyre characteristic.

Two recent examples which do consider the wider state vector are Kiencke and Dai [4], which examines pole-placed observers in both linear and nonlinear forms - though these are stationary - and Ray [5], which develops an extended adaptive Kalman filter. This latter study is similar to the work presented here, but it relies on a higher-order model and larger sensor set including measurement of applied wheel torques.

The Kalman filter is a popular candidate for observers of this type as it utilises available model information along with the measurement set to maximise accuracy. However, to ensure practical real-time operation, the model must remain low-order and time-invariant, whilst retaining a useful level of accuracy; this is a significant constraint given the complex tyre force variations which prevail. Some form of nominal model adaptation, or more formal on-line identification seems inevitable in conjunction with any practically useful Kalman filter.

A preliminary study by the authors [6] illustrates the problem; a bicycle handling model was used as the basis for the filter; $\dot{\mathbf{x}}(t)=\Phi \mathbf{x}(t)+\Gamma \delta(t)$ with $\mathbf{x}(t)=\left[\begin{array}{ll}r & v\end{array}\right]^{T}$ and

$$
\Phi=\left[\begin{array}{cc}
\frac{-b^{2} C_{\alpha f}-c^{2} C_{\alpha r}}{I_{z z} u} & \frac{-b C_{\alpha f}+c C_{\alpha r}}{I_{z z} u} \\
\frac{-b C_{\alpha f}+c C_{\alpha r}-M u^{2}}{M u} & \frac{-C_{\alpha f}-C_{\alpha r}}{M u}
\end{array}\right] \quad \Gamma=\left[\begin{array}{c}
\frac{b C_{\alpha f}}{I_{z z}} \\
\frac{C_{\alpha f}}{M}
\end{array}\right]
$$


where the cornering stiffnesses $\mathrm{C}_{\alpha \mathrm{f}}$ and $\mathrm{C}_{\alpha \mathrm{r}}$ were adapted by a simple recursive least-squares method. Both Kalman filter and adaptation were driven using lateral acceleration measurements, eg at the mass centre,

$$
\dot{y}(t)=\dot{v}(t)+u r(t)
$$

Figure 1 is typical of the findings of that study - although relatively fast adaptation of the $C_{\alpha}$ can be achieved and the dynamic variations are reasonably well tracked, the model order is too low. As a result, significant errors develop at very low frequencies in estimates $\hat{C}_{\alpha f}, \hat{C}_{\alpha r}$ and $\hat{v}$ which can not be corrected without direct measurement of the sideslip. Manipulation of equations (1) and (2) shows that the errors in adapted cornering stiffness $\left(\hat{C}_{\alpha}-C_{\alpha}\right)$ can be explained by steady-state sideslip error $(\hat{v}-v)$ according to :

$$
\hat{C}_{\alpha f}=C_{\alpha f}\left(\frac{v-c r}{\hat{v}-c r}\right), \quad \hat{C}_{\alpha r}=C_{\alpha r}\left(\frac{u \delta-v-b r}{u \delta-\hat{v}-b r}\right) .
$$
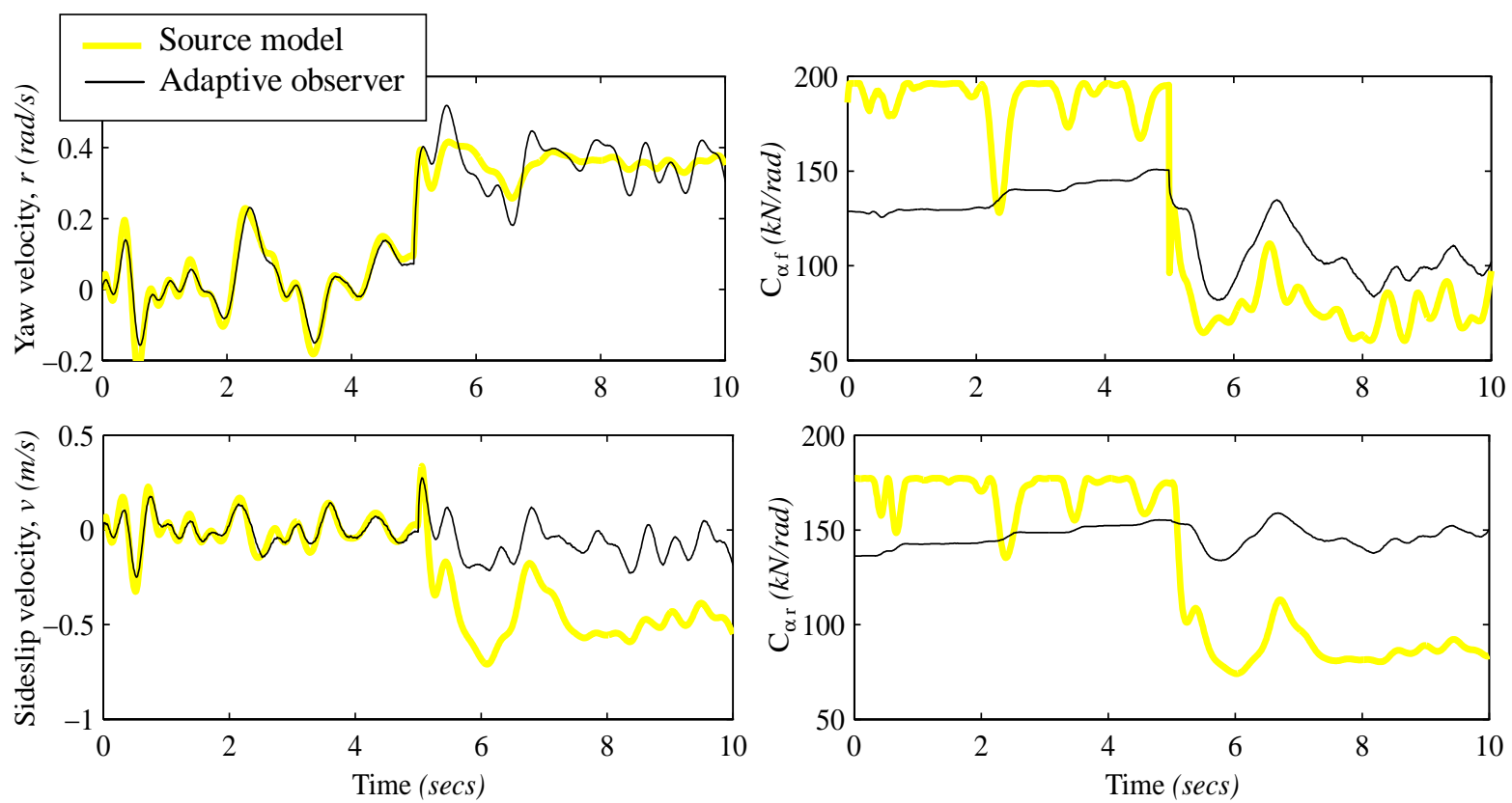

Figure 1 : Illustration of adaptive Kalman filter based on a 'bicycle' handling model

Similar manipulation of alternative extended models shows that inclusion of roll, pitch and or vertical dynamics do not overcome the problem of equation (3), and neither will extension of the tyre model, unless one resorts to the unreasonable assumption of an accurate tyre model without adaptation.

In this study we retain the simple linear type $C_{\alpha}$ model in the Kalman filter, but extend it to include longitudinal dynamics, utilising the small perturbations that steering manouevres induce in forward acceleration, to decouple $C_{\alpha f}$ from $C_{\alpha r}$ and $v$. The philosophy is that steady-state errors can be reduced by adapting the model to closely track a forward acceleration sensor. 
Further enhancements in this simulation study include the use of model nonlinearities in the filter - an extended Kalman filter is prescribed; this has the additional advantage that the adapted parameters $\left(C_{\alpha f}\right.$ and $C_{\alpha r}$ ) can be included in the state vector, so adaptation is implicit within the filter design. Practical viability for production vehicles is also considered throughout the study, in terms of computing requirements, and particularly in the prescription of an inexpensive, reduced sensor set.

Section 2 describes the simulation models, Section 3 details the design algorithm for an extended adaptive filter, and specifies a comparator algorithm. A verification experiment on the influence of correlated noise sources is then given in Section 4. After presentation and discussion of the results in Section 5, a summary of issues for practical viability is provided in Section 6.

\section{SIMULATION}

\subsection{Modelling}

The study is carried out using a reference source model to provide 'true' state trajectories, sensor measurements and also data for simple parameter identifications for the Kalman filter model. This source model simulates full order motion of a rigid vehicle body, with independent suspension freedoms, though vertical suspension modes are suppressed through the assumption of inertialess wheels. A Pacejka tyre model is implemented in both longitudinal and lateral axes, incorporating a friction limiting ellipse.

The precise model equations are omitted here, for brevity, but also - as we will show in Section 4 - because the detail and even to some extent the accuracy of the source data is of secondary importance; the study should reveal similar results for any suitably formulated high order model, or indeed for an actual test vehicle.

For the purposes of the study, we will restrict attention to simulations where the longitudinal dynamic is affected only by steer. Thus the source model is initiated at a constant speed, $25 \mathrm{~m} / \mathrm{s}$, and the (front wheel) drive torque is maintained at a level just sufficient to overcome simulated aerodynamic and rolling friction forces.

This provides a suitable isolated test case for the adaptive observer, and it allows a simplified longitudinal tyre force model to be used in the observer model (though we shall see in Section 5.1 that more representative models could readily be accommodated within the proposed observer design). Figure 2 shows a vehicle plan view, with front axle forces detailed; using small-angle approximations these can be modelled in the SAE axis system as :

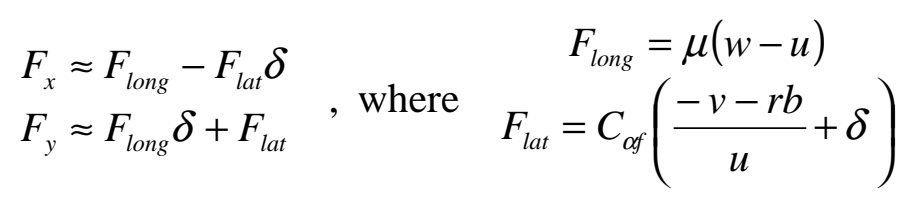




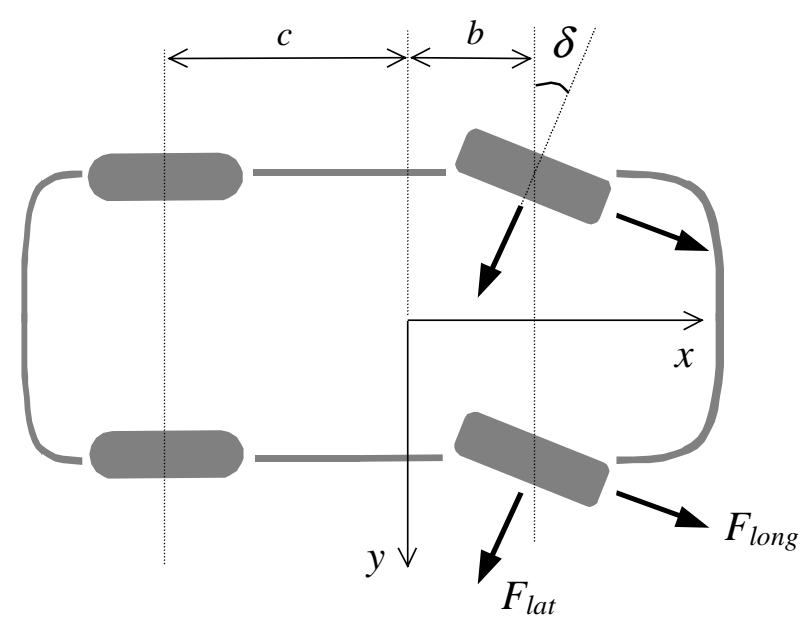

Figure 2: Tyre forces in the observer model

The observer model is then formulated from the popular three degree of freedom yaw/sideslip/roll model described using vehicle centred SAE axes, with a fourth, longitudinal freedom described in terms of the forward speed. The Newton-Euler equations of motion for the five states $[r, v, u, p, \phi]$ are then :

$$
\begin{gathered}
M \dot{v}+M h \dot{p}=\left(\frac{c C_{\alpha r}-b C_{\alpha f}}{u}-M u\right) r-\left(\frac{C_{\alpha f}+C_{\alpha r}}{u}\right) v+\left(C_{\alpha f}+\mu(w-u)\right) \delta \\
M \dot{u}=\frac{b C_{\alpha f} \delta}{u} r+\left(\frac{C_{\alpha f} \delta}{u}+M r\right) v+M h r p+\mu(w-u)-C_{\alpha f} \delta^{2} \\
-I_{x z} \dot{r}+M h \dot{v}+I_{x x} \dot{p}=-M h u r-\left(B_{f}+B_{r}\right) p+\left(M g h-K_{f}+K_{r}\right) \phi \\
I_{z z} \dot{r}-I_{x z} \dot{p}=\left(\frac{-b^{2} C_{\alpha f}-c^{2} C_{\alpha r}}{u}\right) r+\left(\frac{c C_{\alpha r}-b C_{\alpha f}}{u}\right) v+b\left(C_{\alpha f}+\mu(w-u)\right) \delta \\
\dot{\phi}=p
\end{gathered}
$$

Here, both the steer angle $\delta$ and driven wheel speed $w$ are assumed to be available as deterministically known inputs.

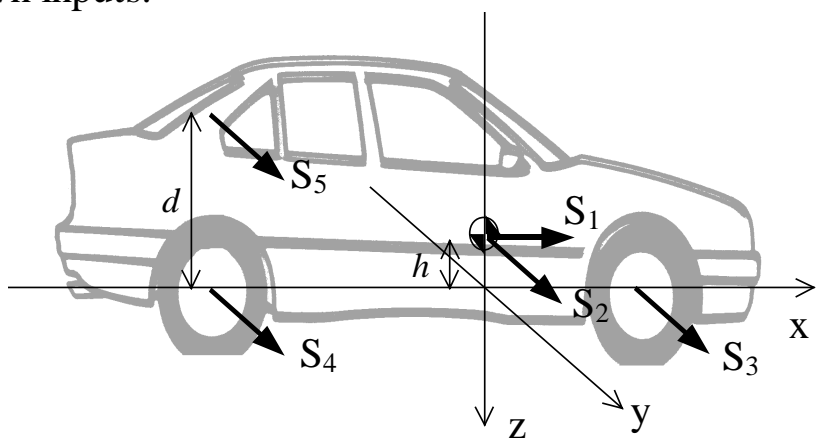

Figure 3: Sensor placement and orientation

Two sensor sets are considered, both exclusively using accelerometers, in the locations given in Figure 3. These are modelled as : 


$$
\begin{gathered}
S_{1}=\dot{u}-r(v+h p)+\varepsilon_{1}, \quad S_{2}=\dot{v}+h \dot{p}+r u+\varepsilon_{2}, \quad S_{3}=\dot{v}+b \dot{r}+r u+\varepsilon_{3} \\
S_{4}=\dot{v}-c \dot{r}+r u+\varepsilon_{4}, \quad S_{5}=\dot{v}+d \dot{p}-c \dot{r}+r u+\varepsilon_{5}
\end{gathered}
$$

where in each case unmodelled measurement noise is simulated using an independent Gaussian white noise signal with equal magnitude on each sensor :

$$
\varepsilon_{i} \propto N\left(0,[2 \rho]^{2}\right)
$$

with $\rho$ used as an amplification factor such that the noise levels are referenced to a nominal RMS lateral acceleration of $2 \mathrm{~m} / \mathrm{s}^{2}$.

Sensor set A employs one measurement for each degree of freedom under consideration, $\mathbf{y}_{A}=$ $\left[S_{1}, S_{2}, S_{3}, S_{4}\right]^{\mathrm{T}}$ whereas set B retains the separate sensor for the longitudinal direction $\left(S_{1}\right)$, but combines detection of lateral, roll and yaw motion in two sensors, $\mathbf{y}_{B}=\left[S_{1}, S_{3}, S_{5}\right]^{\mathrm{T}}$.

\subsection{Parametrisation and Validation}

Parameters for the observer model are obtained from two sources. Inertia and vehicle geometry data are assumed to be known and are thus taken from the source model. Roll stiffness, damping and suitable default values for the tyre parameters $C_{\alpha f}$ and $C_{\alpha r}$ are obtained from a simple identification experiment: source data was simulated on the following input, chosen with a view to feasible vehicle tests, to induce a high level of state dynamic variation within the achievable frequency response envelope of a test driver. :

$$
\delta(t)=N\left(0, \sigma_{\delta}^{2}\right)
$$

$$
\text { bandlimited } 0-5 \mathrm{~Hz}, \sigma_{\delta}=0.017 \mathrm{rad}(1 \text { degree })
$$

Parameter estimates were then made by least-squares regression of this data for a 20 second data batch, sampled at $100 \mathrm{~Hz}$.

Roll stiffness and damping parameters were estimated by regression of the linear-in-parameters equation (7). Cornering stiffness estimates were then obtained by interpreting source model tyre forces and states $F_{t}^{s}, \mathbf{x}^{s}$ in terms of the observer model:

$$
\left(\delta-\frac{v^{s}+b r^{s}}{u^{s}}\right) C_{\alpha f}=F_{t f}^{s}, \quad\left(\frac{-v^{s}+c r^{s}}{u^{s}}\right) C_{\alpha r}=F_{t r}^{s}
$$

Finally, for the simple longitudinal tyre model, the friction coefficient $\mu$ was assigned a nominal value. Due to the nature of the tests undertaken, it can easily be shown that variations from the value chosen have a negligible influence - causing only small changes to steady-state error in the prediction of vehicle forward speed $u$.

To illustrate the resulting observer model accuracy, Figure 4 shows yaw rate and sideslip velocity for a steer input which starts according to equation (12) and then steps to a fixed 
wheel steer angle of $3^{\circ}$; the first section shows good dynamic accuracy, but steady-state errors are high for any significant constant steer angle.
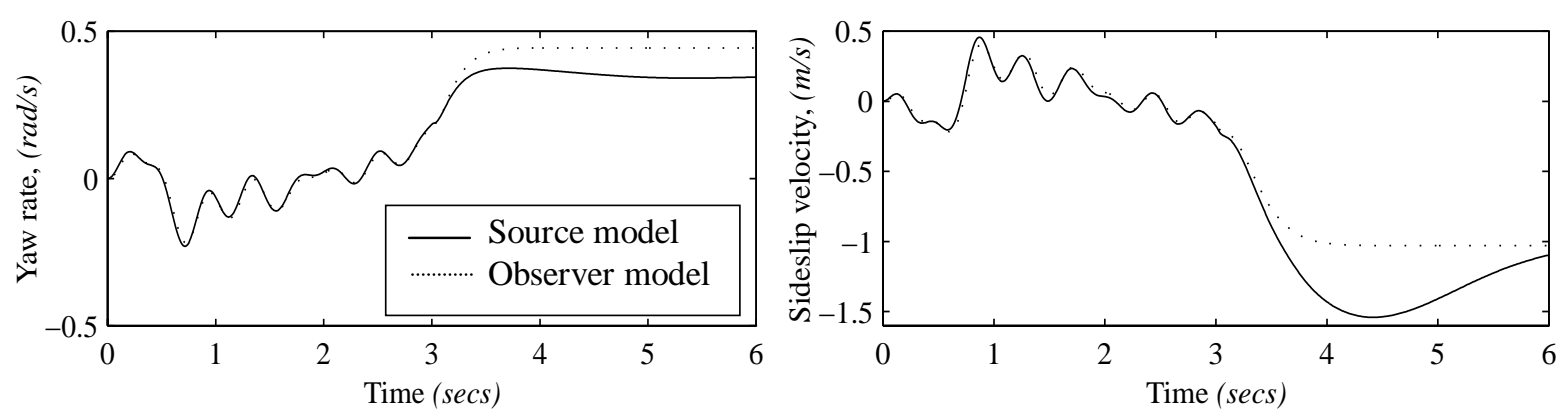

Figure 4: Illustration of source state tracking by the observer model

\section{FORMULATION OF KALMAN FILTERS}

\subsection{Linear Kalman Filter}

As a basis for evaluating the extended Kalman filter we first derive the equivalent linear timeinvariant form. The discrete-time linear model $\mathbf{A}, \mathbf{B}, \mathbf{C}, \mathbf{D}$ is formed from an exponential discretisation of equations (5),(7),(8) and (9) for the reduced state vector $\mathbf{z}=[r, v, p, \phi]^{\mathrm{T}}$, with $u$ assumed constant and taking $\mu=0$. Relating the model to 'true' source states, we can specify process and measurement errors, $\omega_{k}$ and $v_{k}$ respectively

$$
\begin{aligned}
\mathbf{z}_{k+1}^{s} & =\mathbf{A} \mathbf{z}_{k}^{s}+\mathbf{B} \boldsymbol{\delta}_{k}+\omega_{k} \\
\mathbf{y}_{k} & =\mathbf{C} \mathbf{z}_{k}^{s}+\mathbf{D} \boldsymbol{\delta}_{k}+v_{k}
\end{aligned}
$$

and an optimal filter can easily be derived, provided the following expectations $E($ ) apply :

$$
\begin{aligned}
& E\left(\omega_{k}\right)=\mathbf{0}, \quad E\left(v_{k}\right)=\mathbf{0}, \quad \forall k \\
& E\left(\omega_{i} \omega_{j}^{T}\right)=\mathbf{0}, \quad E\left(v_{i} v_{j}^{T}\right)=\mathbf{0}, \quad \forall(i \neq j)
\end{aligned}
$$

The simplest form of the Kalman filter also assumes $E\left(\omega_{k} v_{k}^{T}\right)=\mathbf{0}, \forall k$ but this can not be expected for either sensor set here; for accelerometers the sensor model $\mathbf{C}, \mathbf{D}$ correlates with $\mathbf{A}, \mathbf{B}$, and this ensures that $\omega_{\mathrm{k}}$ and $v_{\mathrm{k}}$ share a common modelling error component. The Kalman filter is thus specified in its most general form (eg from [7]),

$$
\hat{\mathbf{z}}_{k+1}=(\mathbf{A}-\mathbf{K C}) \hat{\mathbf{z}}_{k}+(\mathbf{B}-\mathbf{K D}) \boldsymbol{\delta}_{k}+\mathbf{K} \mathbf{y}_{k}
$$

with the (time-invariant) gain matrix $\mathbf{K}$ found through calculation of the anticipated state error covariance matrix $\mathbf{P}$, which is the solution to the algebraic Riccati equation :

$$
\begin{aligned}
& \mathbf{P}=\mathbf{G P G}^{\mathrm{T}}-\mathbf{G P C}^{\mathrm{T}}\left[\mathbf{C P C}^{\mathrm{T}}+\mathbf{R}\right]^{-1} \mathbf{C P G}^{\mathrm{T}}+\overline{\mathbf{Q}} \\
& \mathbf{K}=\left(\mathbf{A P C}^{\mathrm{T}}+\mathbf{S}\right)\left(\mathbf{C P C}^{\mathrm{T}}+\mathbf{R}\right)^{-1}
\end{aligned}
$$


where

$$
\mathbf{G}=\mathbf{A}-\mathbf{S R}^{-1} \mathbf{C}
$$

$$
\overline{\mathbf{Q}}=\mathbf{Q}-\mathbf{S R}^{-\mathbf{1}} \mathbf{S}^{\mathbf{T}}
$$

and

$$
\mathbf{e}_{k}=\left[\begin{array}{c}
\boldsymbol{\omega}_{k} \\
\boldsymbol{v}_{k}
\end{array}\right], \quad E\left(\mathbf{e}_{k} \mathbf{e}_{k}^{\mathbf{T}}\right)=\left[\begin{array}{cc}
\mathbf{Q} & \mathbf{S} \\
\mathbf{S}^{\mathbf{T}} & \mathbf{R}
\end{array}\right]
$$

\subsection{Extended Kalman Filter}

The extended filter employs the fully nonlinear model of equations (5) - (9), which can be written for continuous state propagation, and sampled sensor data in the general form,

$$
\begin{aligned}
& \dot{\mathbf{x}}^{s}(t)=\mathbf{f}\left(\mathbf{x}^{s}(t)\right)+\omega(t) \\
& \mathbf{y}_{k}=\mathbf{h}\left(\mathbf{x}_{k}^{s}\right)+v_{k}
\end{aligned}
$$

Clearly $\mathbf{f}()$ and $\mathbf{h}()$ also depend on $\delta$ and $w$ here; as deterministically known inputs, these are readily incorporated in the algorithm, but in the derivation below they are not referred to explicitly, to avoid over-complication of the notation.

Gelb et al [7] propose estimation of $\hat{\mathbf{x}}$ by the use of a truncated Taylor series for $\mathbf{f}(\mathbf{x}(t))$ in the continuous model and state error covariance matrix estimate. The observer is then derived in a continuous-discrete form, using continuous model propagation combined with sampled measurements which provide discrete state corrections. The final form is then fully discretised to a practicable form.

Assuming time-invariant noise covariance matrices, the resulting algorithm provides continuous propagation models :

$$
\begin{gathered}
\dot{\hat{\mathbf{x}}}(t)=\mathbf{f}(\hat{\mathbf{x}}(t)) \\
\dot{\mathbf{P}}(t)=\mathbf{F}(\hat{\mathbf{x}}(t)) \mathbf{P}(t)+\mathbf{P}(t) \mathbf{F}^{\mathrm{T}}(\hat{\mathbf{x}}(t))+\mathbf{Q}
\end{gathered}
$$

with the sensors providing filter and state corrections at each discrete time step - improving the variable estimates from the (-) to (+) form :

$$
\begin{aligned}
& \hat{\mathbf{x}}_{k}(+)=\hat{\mathbf{x}}_{k}(-)+\mathbf{K}_{k}\left[\mathbf{y}_{k}-\mathbf{h}\left(\hat{\mathbf{x}}_{k}(-)\right)\right] \\
& \mathbf{P}_{k}(+)=\left[\mathbf{I}-\mathbf{K}_{k} \mathbf{H}\left(\hat{\mathbf{x}}_{k}(-)\right)\right] \mathbf{P}_{k}(-)
\end{aligned}
$$

where

$$
\mathbf{K}_{k}=\mathbf{P}_{k}(-) \mathbf{H}^{\mathrm{T}}\left(\hat{\mathbf{x}}_{k}(-)\right)\left[\mathbf{H}\left(\hat{\mathbf{x}}_{k}(-)\right) \mathbf{P}_{k}(-) \mathbf{H}^{\mathrm{T}}\left(\hat{\mathbf{x}}_{k}(-)\right)+\mathbf{R}\right]^{-1}
$$

In the above, matrices $\mathbf{F}$ and $\mathbf{H}$ are Jacobians resulting from the Taylor series expansions, evaluated at the state estimates :

$$
\begin{aligned}
& \mathbf{F}(\hat{\mathbf{x}}(t))=\left.\frac{\partial \mathbf{f}(\mathbf{x}(t))}{\partial \mathbf{x}(t)}\right|_{\mathbf{x}(t)=\hat{\mathbf{x}}(t)} \\
& \mathbf{H}(\hat{\mathbf{x}}(t))=\left.\frac{\partial \mathbf{h}(\mathbf{x}(t))}{\partial \mathbf{x}(t)}\right|_{\mathbf{x}(t)=\hat{\mathbf{x}}(t)}
\end{aligned}
$$


Now, as the quoted formulation neglects process and measurement noise correlation, $E\left(\omega(t) v_{k}^{T}\right)=\mathbf{0}$, we require a modified form. This is achieved as for the linear Kalman filter theory, by the use of a Lagrange multiplier; if we redefine $\dot{\mathbf{x}}(t)$ from equations (19) such that

$$
\dot{\mathbf{x}}^{s}(t)=\mathbf{f}\left(\mathbf{x}^{s}(t)\right)+\omega(t)+\mathbf{S R}^{-1}\left\{\mathbf{y}_{k}-\mathbf{h}\left(\mathbf{x}_{k}^{s}\right)-v_{k}\right\}
$$

With $\mathbf{S}$ defined as in Section 3.1, we derive

where

$$
\begin{array}{r}
\dot{\mathbf{x}}^{s}(t)=\mathbf{f}^{*}\left(\mathbf{x}^{s}(t)\right)+\omega^{*}(t) \\
\mathbf{f}^{*}\left(\mathbf{x}^{s}(t)\right)=\mathbf{f}\left(\mathbf{x}^{s}(t)\right)+\mathbf{S R}^{-1}\left[\mathbf{y}_{k}-\mathbf{h}\left(\mathbf{x}_{k}^{s}\right)\right] \\
\omega^{*}(t)=\omega(t)+\mathbf{S R}^{-1} \mathbf{v}_{k}
\end{array}
$$

and it is easily verified that $E\left(\omega^{*}(t) v_{k}^{T}\right)=\mathbf{0}$.

Since $\mathbf{y}_{k}$ is taken as a deterministic input in equation (24), we can write

$$
\mathbf{F}^{*}(\hat{\mathbf{x}}(t))=\mathbf{F}(\hat{\mathbf{x}}(t))-\mathbf{S} \mathbf{R}^{-1} \mathbf{H}(\hat{\mathbf{x}}(t))
$$

and also define an equivalent process covariance,

$$
E\left(\omega^{*}(t) \omega^{* T}(t)\right)=\mathbf{Q}^{*}=\mathbf{Q}-\mathbf{S R}^{-\mathbf{1}} \mathbf{S}^{\mathbf{T}}
$$

Assimilating these modifications, and using the simplest Euler integration for numerical implementation, the final algorithm is given for sample time $T$, by

$$
\begin{gathered}
\mathbf{K}_{k}=\mathbf{P}_{k} \mathbf{H}^{\mathbf{T}}\left(\hat{\mathbf{x}}_{k}\right)\left[\mathbf{H}\left(\hat{\mathbf{x}}_{k}\right) \mathbf{P}_{k} \mathbf{H}^{\mathbf{T}}\left(\hat{\mathbf{x}}_{k}\right)+\mathbf{R}\right]^{-1} \\
\mathbf{P}_{k}^{*}=\left[\mathbf{I}-\mathbf{K}_{k} \mathbf{H}\left(\hat{\mathbf{x}}_{k}\right)\right] \mathbf{P}_{k} \\
\mathbf{P}_{k+1}=\mathbf{P}_{k}^{*}+T\left[\mathbf{F}^{*}\left(\hat{\mathbf{x}}_{k}\right) \mathbf{P}_{k}^{*}+\mathbf{P}_{k}^{*} \mathbf{F}^{* \mathbf{T}}\left(\hat{\mathbf{x}}_{k}\right)+\mathbf{Q}^{*}\right] \\
\hat{\mathbf{x}}_{k+1}=\hat{\mathbf{x}}_{k}+\mathbf{K}_{k}\left(\mathbf{y}_{k}-\mathbf{h}\left(\hat{\mathbf{x}}_{k}\right)\right)+T\left[\mathbf{f}\left(\hat{\mathbf{x}}_{k}\right)+\mathbf{S R}^{-\mathbf{1}}\left(\mathbf{y}_{k}-\mathbf{h}\left(\hat{\mathbf{x}}_{k}\right)\right)\right]
\end{gathered}
$$

Equations (25) to (28) constitute the full update set required for on-line evaluation. The sequence presents a computational burden in the inverse calculation for $\mathbf{K}_{k}$, but this is not deemed excessive for the model order considered - even for relatively high sample rates given modern computing capacity.

\section{FIXED MODEL OBSERVER VERIFICATION}

A first simulation experiment is conducted to illustrate the effect of model and sensor errors which do or do not conform to the ideal assumptions of the theory outlined above.

As a simple illustration of tracking performance, the estimation of sideslip velocity is examined in response to a $3^{\circ}$ step-steer manoeuvre, for both the linear and extended Kalman filters, using 
sensor set A. A grid of four different design / test conditions is examined, and the results are illustrated in Figure 5.

The upper plots show results from observers which were designed using the general Kalman filter algorithms given in Section 3, with the noise cross correlation matrix $\mathbf{S}$ included. In the lower plots, the simplified forms are considered, with $\mathbf{S}=0$ for both algorithms.

For the right hand plots (b), the simulated source states are used directly, such that $\omega$ and $v$ correctly reflect modelling errors, and a nominal setting $(\rho=0.2)$ is used for additional sensor noise. On the left, plots (a) show the idealised noise condition, for which the filters are strictly optimal. In this case, the 'source' states were simulated directly from equations (14) or (19) rather than from the source model, with independent Gaussian white noise series applied to each state $i$,

$$
\omega_{i}(t) \propto N\left(0, \sigma_{i}\right)
$$

where $\sigma_{i}$ was chosen to ensure equal $\operatorname{RMS}\left(\omega_{i}(t)\right)$ in plots (a) and (b). Cross correlated errors were applied to the sensors for this case using $v=\mathbf{U} \omega$, where $\mathbf{U}$ is appropriately dimensioned, with uniformly distributed random elements in the range $(0,1)$.

For each of the tests, to maximise performance potential the noise covariance matrices were then estimated from the $N$ data points of the available series $\omega$ and $v$ :

$$
\left[\begin{array}{ll}
\mathbf{Q} & \mathbf{S} \\
\mathbf{S}^{\mathbf{T}} & \mathbf{R}
\end{array}\right]=\frac{\left(\mathbf{e e}^{T}\right)}{N-1}, \quad \mathbf{e}=\left[\begin{array}{l}
\omega \\
v
\end{array}\right]
$$

(a) White Noise Model Errors
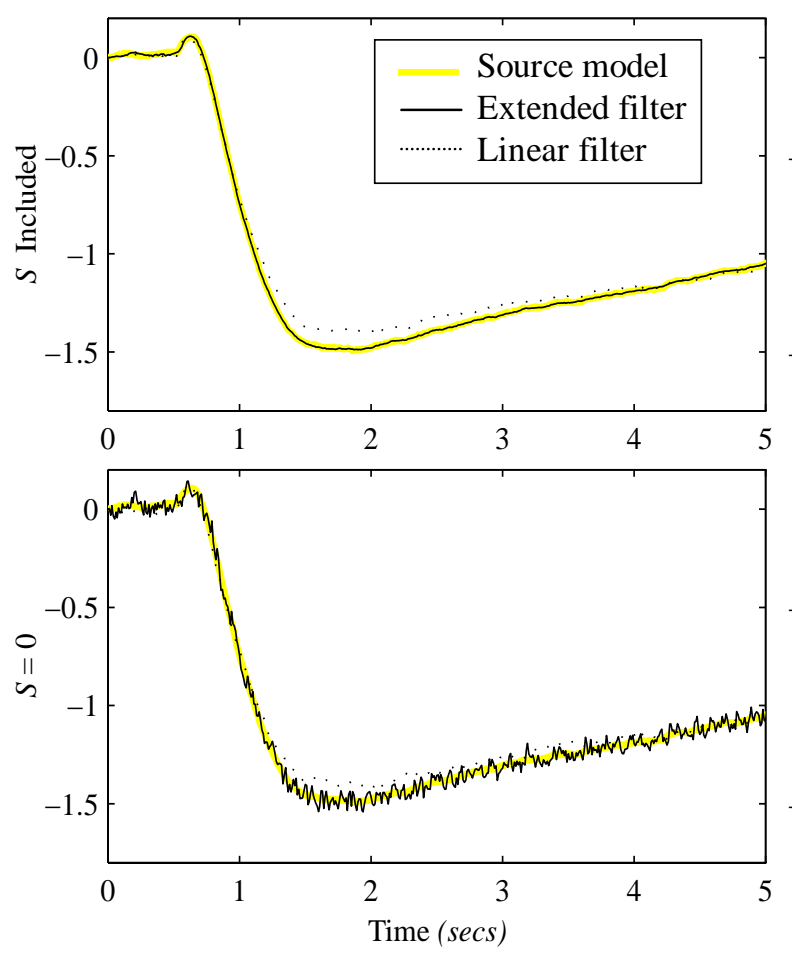

(b) Source Model Errors
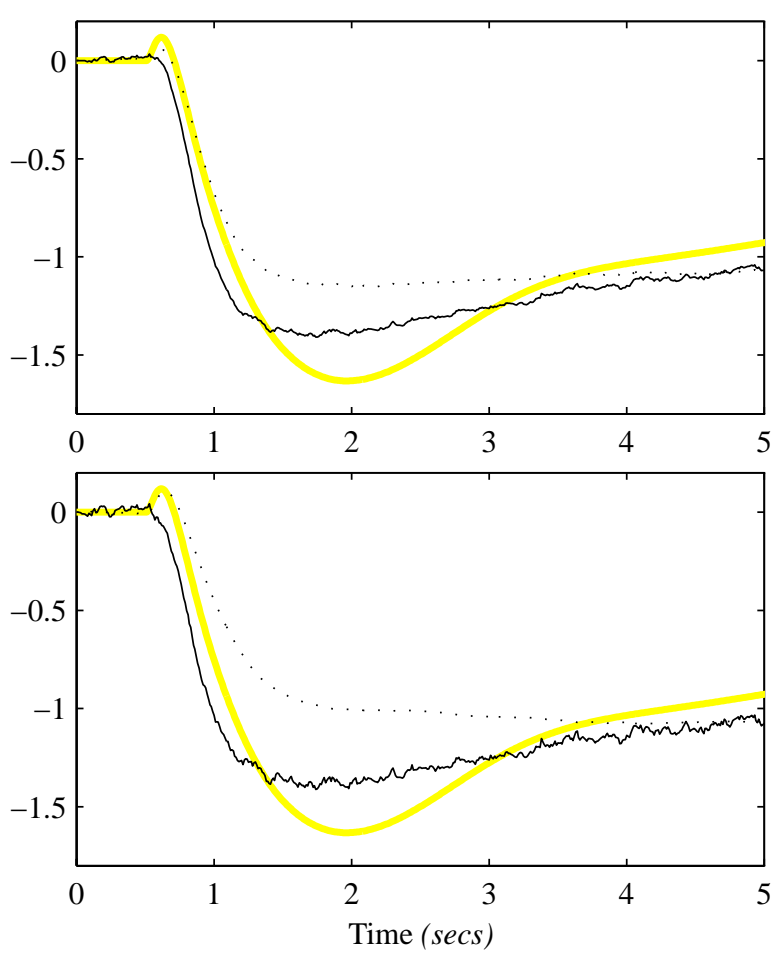

Figure 5: The effect of cross-correlated error sources on sideslip velocity estimations 
Plots (a) provide a good illustration of the effect of the $S$ matrix; in both filters there is a significant reduction of errors, particularly at high frequencies. Some performance advantage of the nonlinear algorithm is also seen here, although this is clearer in plots (b).

Plots (b) reflect a fundamental and practical problem with Kalman filters. Although improvements are still evident for $\mathbf{S}$ matrix inclusion, the far greater source of discrepancy is due to model errors similar to those seen in Figure (4) (for example, the linear filter does not track the gradually decreasing sideslip which is caused by decreasing forward speed). The design assumption, that $E\left(\omega^{*}(t) \mathbf{x}_{k}^{\mathbf{T}}\right)=\mathbf{0}$ is not valid here, so in practice, the Kalman filter will be sub-optimal. One method of improving Kalman filter performance in these cases is to increase $\mathbf{Q}$ to reflect the significant low frequency bias in $\omega$. This will not be further examined here, but has been shown to give improved results for vertical suspension observers in [8]. The approach we adopt here is to adapt the observer model, with the aim of reducing the low frequency model errors that correlate with state estimates.

\section{EXTENDED ADAPTIVE KALMAN FILTER}

\subsection{Model Adaptation}

Given the general form for $\mathbf{f}(\mathbf{x}(t))$, the model can readily be specified in terms of an extended state vector $\chi(\mathrm{t})$ :

$$
\dot{\chi}(t)=\left[\begin{array}{c}
\dot{\mathbf{x}}(t) \\
\dot{C}_{\alpha f}(t) \\
\dot{C}_{\alpha r}(t)
\end{array}\right]=\left[\begin{array}{c}
\mathbf{f}(\mathbf{x}(t)) \\
\mathbf{f}_{\alpha}(\mathbf{x}(t))
\end{array}\right]+\left[\begin{array}{c}
\omega(t) \\
\omega_{\alpha}(t)
\end{array}\right]
$$

This provides for some predictive model for the $C_{\alpha}$, but we can alternatively choose $\mathbf{f}_{\alpha}(\mathbf{x}(t))=\mathbf{0}$ thereby assuming all variation in the cornering stiffness to be noise-generated. Given the significant and rapid variations which actually occur in $C_{\alpha}$, this assumption will lead to very large values in the corresponding elements of $\mathbf{Q}$ and $\mathbf{S}$. Now, through equations (25) (27) the Kalman filter attempts to balance the sensor error feedback $\left(\mathbf{K}_{k}\right)$ and propagation $(T)$ terms in equation (28), to reflect the relative magnitudes of model and measurement error. Given very large modelling error for the $C_{\alpha}, \mathbf{K}_{k}$ provides high gain feedback of sensor error to these states, and hence rapid adaptation. (This is equivalent to the Kalman filter 'trusting' the sensor information much more than the model information, as the latter is known to be poor.)

In practice the adaptation resulting from $\mathbf{f}_{\alpha}(\mathbf{x}(t))=\mathbf{0}$ is too fast, giving an unstable observer for all cases examined. The effect is therefore moderated, by incorporating a tuning parameter $\lambda$ to modify $\mathbf{Q}$ and $\mathbf{R}$ :

$$
\begin{gathered}
\mathbf{Q}_{\lambda}=\mathbf{I}_{\lambda} \mathbf{Q} \mathbf{I}_{\lambda} \\
\mathbf{S}_{\lambda}=\mathbf{I}_{\lambda} \mathbf{S}
\end{gathered}, \quad \mathbf{I}_{\lambda}=\left[\begin{array}{ccc}
\mathbf{I}_{5 \times 5} & 0 & 0 \\
0 & \lambda & 0 \\
0 & 0 & \lambda
\end{array}\right], \quad \lambda \in[0,1]
$$

Choosing a lower value for $\lambda$ reduces the perceived error, and hence the adaptation rate - the invariant model for $C_{\alpha}$ being 'trusted' more. 


\subsection{Simulation Results}

Figure 6 compares state and sensor trajectories for both observers (using sensor set A), against source model data. The figure also includes a dynamic comparison of the adapted cornering stiffness parameters - plots (f) and (g) - where source data has been calculated from the quotient of instantaneous tyre forces and slip angles. The input is a lane-change manouevre followed by two rapid steer events, simulated using step changes passed through a first order filter; this was chosen to illustrate transient dynamic effects, and also test the observer's steady-state response at two different steer angles - see plot 6(j).

For these results and for those in Figure 7, the noise and system models are kept constant $-\mathbf{Q}$, $\mathbf{R}$ and $\mathbf{S}$ being determined as for Fig 5(b), with $\rho=0.2$. The adaptation parameter has been tuned approximately, to $\lambda=5 \times 10^{-4}$.

The extended adaptive Kalman filter (EAKF) is clearly more susceptible to noise than the standard filter (SKF), but state tracking is generally better - particularly for the sideslip velocity. In spite of the high levels of noise on the longitudinal acceleration - plot (h) - small steady-state changes in this signal are sufficient to induce appropriate adaptation in the $C_{\alpha}$. This influence is seen particularly well immediately after the step steer at 2.5 seconds. Plot (h) shows that the filter takes approximately two seconds to adjust to the new steady-state longitudinal acceleration; during this time both $C_{\alpha}$ are over-estimated, and this causes corresponding errors in the sideslip velocity, in plot (b).

Figure (6) shows that the new observer is successful for the full sensor set A, but the same test using set B actually achieves better performance, despite having fewer sensors. Table 1 shows RMS tracking errors as a percentage of the RMS state, for both EAKF sensor configurations compared with SKF.

\begin{tabular}{c|c|c|c|c|c|}
$\%$ & $r$ & $v$ & $u$ & $p$ & $\phi$ \\
\hline SKF & 11.0 & 40.7 & 0.4 & 21.8 & 10.4 \\
EAKF (Set A) & 7.4 & 31.8 & 0.3 & 52.5 & 4.6 \\
EAKF (Set B) & 3.8 & 18.5 & 0.3 & 40.3 & 3.7 \\
\hline
\end{tabular}

Table 1 : Performance comparison - RMS error as a percentage of signal

The only poor result for either EAKF is in estimation of $p$, where high RMS errors can be seen from plot 6(d) to be due to noise sensitivity; in spite of this, the plot shows that transients are well tracked, and the related roll angle estimation is consistently good. Such high sensitivity to noise can generally be tuned out at the Kalman filter design stage by appropriate adjustment of the noise matrices, so poor results for $\hat{p}$ are not considered to be a significant flaw of the new observer. 

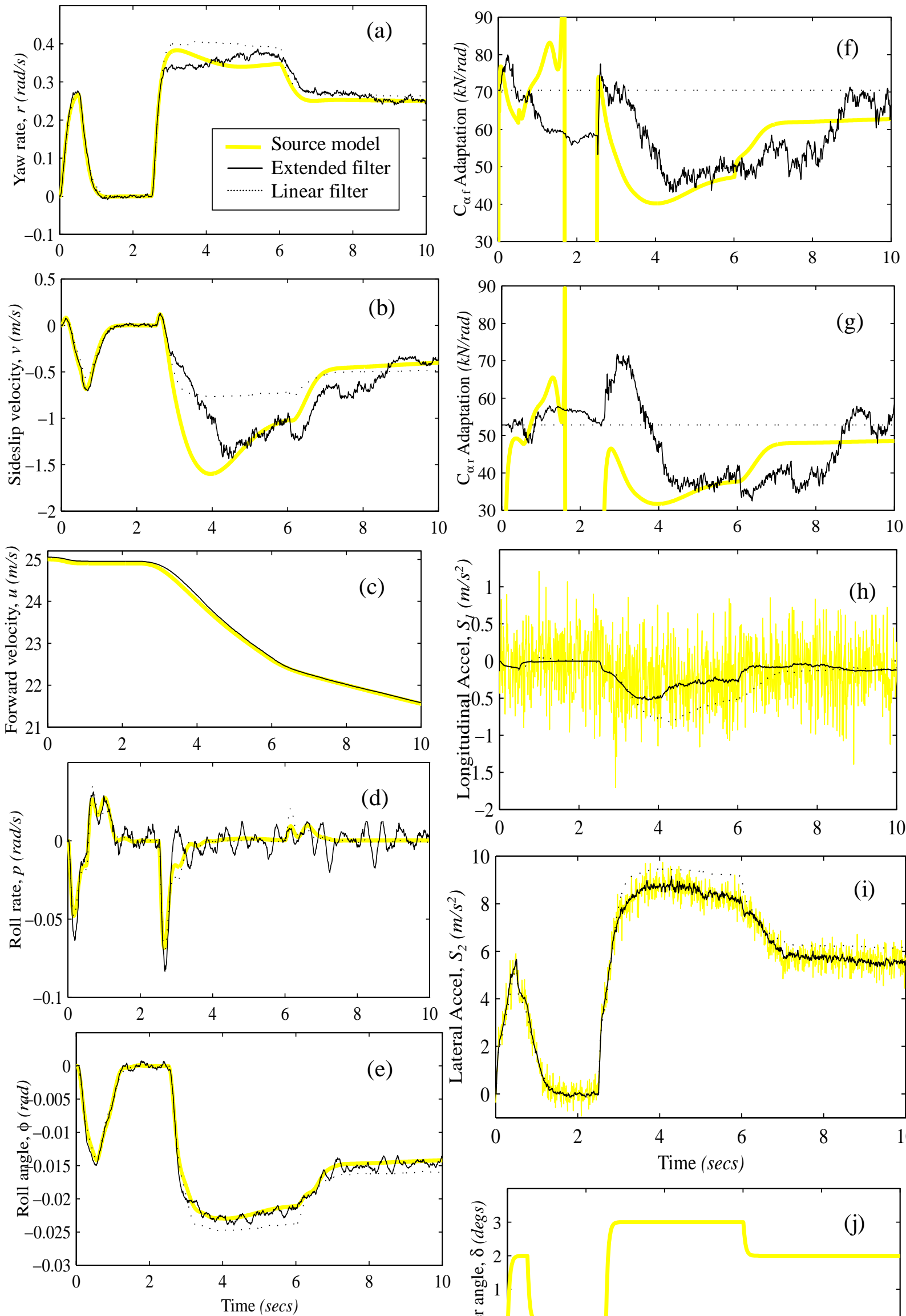

Figure 6: Performance comparison between linear and extended adaptive Kalman filters - sensor set A
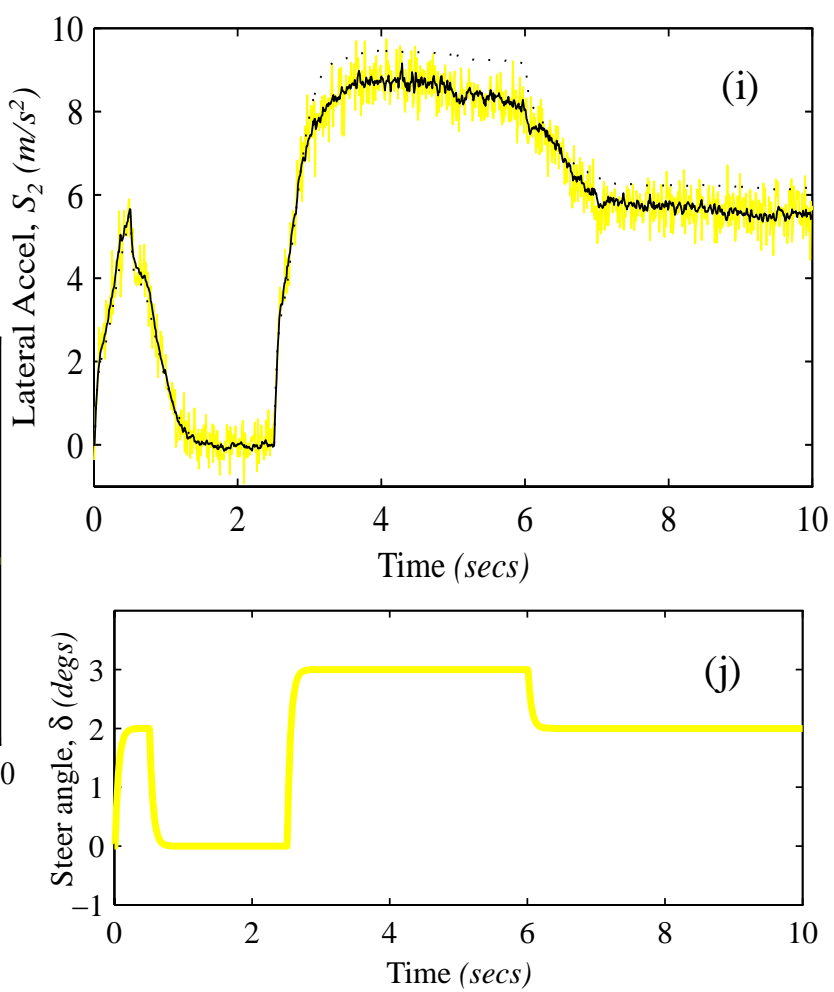
The new filter's performance is clearly reliant on the speed of adaptation following an input transient, and this can be directly influenced by varying $\lambda$. Figure 7 shows a reduced set of the key states for the same test as in Figure 6, using sensor set B, and here we examine the effect of a significant change in $\lambda$.

$\lambda$ is not a sensitive parameter in the algorithm, but magnifying it by a factor of ten causes high adaptation, which results in excellent tracking initially, during the transient around $t=3$ seconds, but quickly starts to become unstable. After $t=6$ seconds, the decreased longitudinal component of acceleration reduces the model conditioning such as to cause rapid destabilisation. Interestingly, this only greatly disrupts $\hat{v}$ - inducing a model error similar to that in equation (3). The effect on $\hat{v}$ of too low a choice for $\lambda$ (not illustrated) is similar, but due instead to slower adaptation, although low $\lambda$ does has the minor redeeming feature of reduced noise transmission.
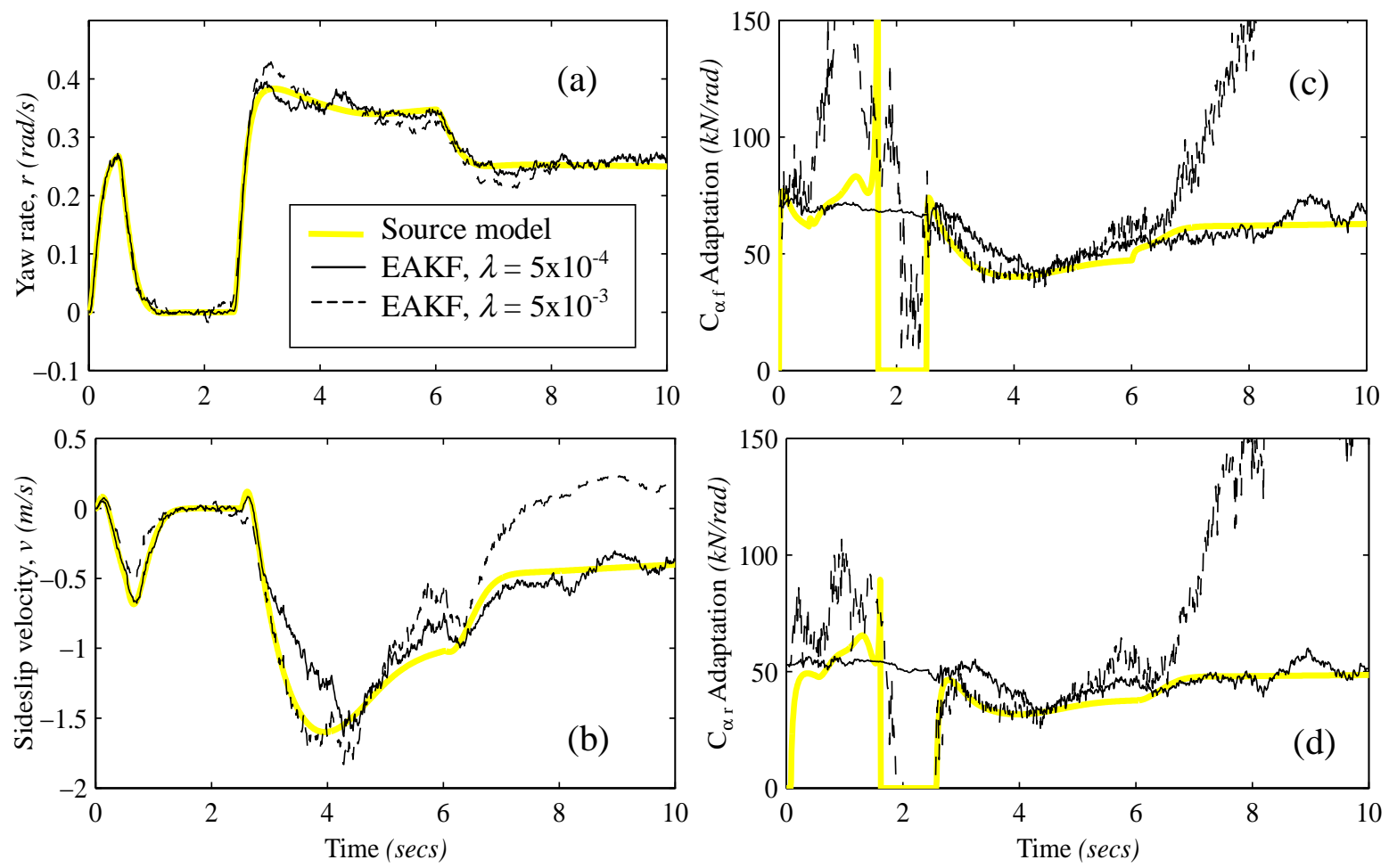

Figure 7 : Extended adaptive Kalman filter performance for two choices of $\lambda$-sensor set $B$

Plot 7(c) also shows the effect of undetermined $\hat{C}_{\alpha}$ where $\delta=0$ between 1 and 2.5 seconds. To prevent inappropriate variation of $\hat{C}_{\alpha}$ some dynamic variation of $\lambda$ would be valid, particularly as errors increase with $\delta$. Indeed improvements can be seen by setting $\lambda$ proportional to $\delta$, but the need for such parameter 'fiddling' naturally leads to the more sensible conclusion that a nonlinear tyre model may be warranted as part of a further development of the observer.

Finally, a clear demonstration of the sensitivity of $\lambda$ to various measurement noise conditions is given by the surface plot of Figure 8. Here EAKF (set B) performance is quantified simply by 
the RMS error to signal ratio in $\hat{v}$ (capped at 40\%), as $\rho$ and $\lambda$ vary, where for each simulation the noise matrices are regenerated in line with the changes in $\rho$. If $\lambda$ is chosen in the correct order of magnitude, the new algorithm is capable of reasonable noise rejection in excess of $\rho=$ 0.4 .

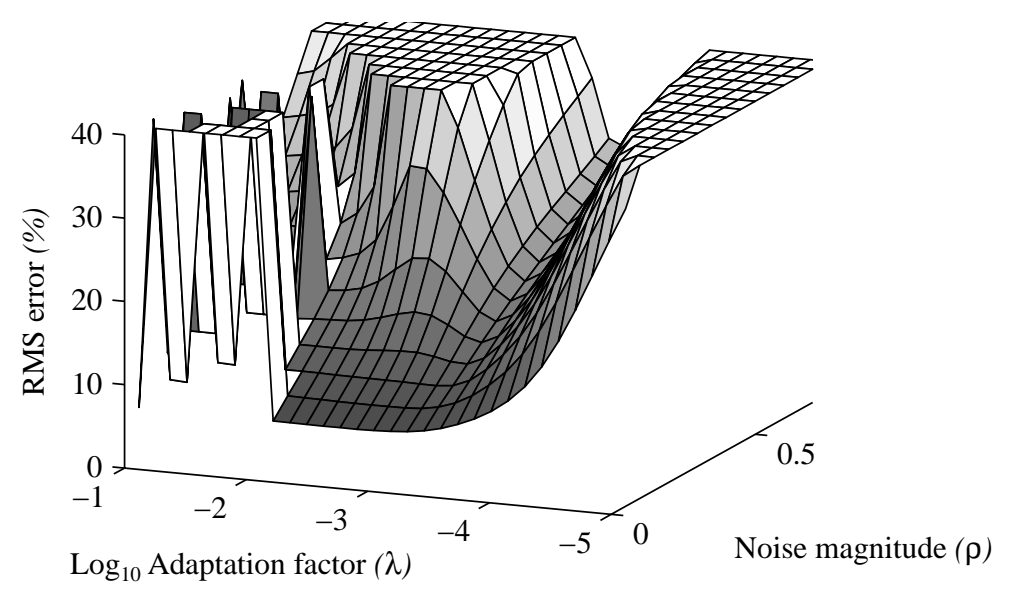

Figure 8: Surface plot illustrating observer sensitivity to parameter and test condition variation

\section{PRACTICAL VIABILITY}

Although these results show promise for the EAKF, there are a number of issues which would need to be resolved before any practically viable observer could be developed. A minor example is the parameter identification, which could not be conducted exactly as specified; however, suitable alternative solutions can readily be found for this problem (eg by transfer function estimation).

The most significant issues are related to over-simplification of the tyre behaviour in the observer model. Although adaptation is successful, the use of a linear model makes the process too slow to provide accurate transient data which may be required for control. Also, we have not examined longitudinal tyre modes which will be critical given the dependence of the algorithm on longitudinal acceleration.

On the other hand, $\mathbf{f}_{\alpha}(\mathbf{x}(t))$ could easily accommodate a nonlinear differentiable tyre model; some simplification of the magic tyre formula in both axes seems a likely candidate. The critical benefit of such an extended model would be that adaptation could graduate from tracking the tyre force to the estimation of adhesion - although this would require an accurate cross-compatibility between the longitudinal and lateral tyre models. Pragmatically, this crosscompatibility is likely to be key, given the need to differentiate between changes in acceleration which are due to engine torque and braking, and those due to the longitudinal effects of steer.

One disadvantage of an extended tyre model would be an increase in the on-line computational burden. Though this is likely to be a minor issue, it is possible to linearise the extended Taylorseries based filter about an operating point - thereby allowing off-line calculation of the Kalman gains $\mathbf{K}_{k}$. This may provide a practicable alternative if computation capacity is 
insufficient to accommodate the matrix inversion on-line, particularly if several pre-calculated observers were gain-scheduled.

Finally, the estimation of noise covariance matrices is idealised in the study. Unfortunately, practical estimation is almost impossible without elaborate instrumentation of a test vehicle, but even then, noise measurements may not provide the most successful covariance matrices. Bearing in mind the findings from Figure 4, and the experience of $\hat{p}$ in Figure 6, the best solution will probably be achieved through some nominal assignation of the covariances, and subsequent manual or automated on-line tuning - perhaps using recent research methods for intelligent 'learning' [9].

\section{CONCLUSIONS}

A nonlinear Extended Adaptive Kalman Filter (EAKF) has been proposed for the on-line estimation of vehicle handling dynamic states. Although it has been shown that this application inevitably results in a suboptimal Kalman filter, the simulation results suggest that such a filter may form the basis of a practical observer. For the steering tests conducted, the EAKF successfully adapts to varying tyre characteristics, and hence provides improved performance over a comparable conventional Kalman filter.

\section{REFERENCES}

1. Furukawa, Y. and Abe, M.: Advanced Chassis Control Systems for Vehicle Handling and Active Safety. Vehicle System Dynamics. Vol 28 (1997), pp. 59-86.

2. Nagai, M., Hirano Y. and Yamanaka, S.: Integrated Control Law of Active Rear Wheel Steering and Direct Yaw Moment Control. Proc. $3^{\text {rd }}$ International Symposium on Advanced Vehicle Control (AVEC), Aachen, 1996, pp. 451-469.

3. Alberti, V. and Babbel, E.: Improved Driving Stability by Active Braking of the Individual Wheels. Proc. $3^{\text {rd }}$ International Symposium on Advanced Vehicle Control (AVEC), Aachen, 1996, pp. 717-732.

4. Kiencke, U. and Daiss, A.: Observation of Lateral Vehicle Dynamics. Control Engineering Practice. Vol 5, No 8, pp. 1145-1150.

5. Ray, L.R.: Nonlinear Tire Force Estimation and Road Friction Identification: Simulation and Practice. Automatica. Vol 33, No 10, pp. 1819-1833.

6. Best, M.C. and Gordon, T.J.: Real-time State Estimation of Vehicle Handling Dynamics using an Adaptive Kalman Filter. Proc. $4^{\text {th }}$ International Symposium on Advanced Vehicle Control (AVEC), Nagoya, Japan, Sept. 1998, pp. 183-188.

7. Gelb, A.: Applied Optimal Estimation. MIT Press, Cambridge Mass. 1974. 
8. Best, M.C.: On the Modelling Requirements for the Practical Implementation of Advanced Vehicle Suspension Control. Doctoral thesis, Loughborough University, 1995.

9. Howell, M.N., Frost, G.P., Gordon, T.J. and Wu, Q.H.: Continuous Action Reinforcement Learning Applied to Vehicle Suspension Control. Mechatronics, Vol 7(3), 1997, pp. 263-276. 